\title{
Nutritional Anemia Status in Adolescent Girls in Rural Schools of Raipur, India
}

Suprava Patel ${ }^{1 *}$, Puja Dhuppar ${ }^{2}$ and Bhattar $\mathrm{A}^{2}$

${ }^{1}$ Department of Biochemistry, All India Institute of Medical Sciences, Raipur, Chhattisgarh, India

${ }^{2}$ Balgopal Children Hospital and Research Center, Raipur, Chhattisgarh, India

\begin{abstract}
Introduction: Nutritional deficiency, especially iron deficiency is the most common etiological factor for anemia, particularly in adolescent girls when the requirement for iron increases tremendously to physiological need.

Aim: This study not only would highlight the burden of nutritional anemia in them, but also it would aid in drawing recommendations and rendering suggestions to assess and maneuver the existing schemes. The aim was to estimate the prevalence of anemia among school going girls to associate anemia to their demographic profile and nutritional status.
\end{abstract}

Materials and methods: This was a community based cross sectional observational study. The demographic profile was collected followed by estimation of Iron profile and Vitamin B12 levels estimation among 382 adolescent girls aged 10 to 18 years, in schools at rural area of Raipur district, India.

Results: Iron and vitamin B12 deficiency was present in $11 \%$ and $58 \%$ respectively. Statistically significant association was observed between severity of anemia serum ferritin $(p<0.01)$ but not with serum vitamin B12 levels.

Conclusion: Anemia in adolescent girls was found to be very common. Moreover, Vitamin B12 deficiency was more common than iron deficiency in adolescent girls. There is need to shift the focus from old paradigm of iron deficiency anemia to other forms of anemia basically vitamin B12 deficiency anemia, as observed from this study.

Keywords: Adolescent girls; Anemia; Iron deficiency; Vitamin B12 deficiency

\section{Introduction}

Anemia is defined by World Health Organization (WHO) as low blood hemoglobin concentration, $<12 \mathrm{gm} / \mathrm{dl}$, and has been regarded as public health problem in both developed and developing countries [1]. In-spite of successful implementation of National Nutritional Anemia Control Programme (NNACP) and Iron Folic acid supplementation (IFA) - National Nutritional Anemia Prophylaxis programme, the burden of anemia in surprisingly large enough in Indian children, particularly in adolescent girls [2]. As per the National Rural Health Mission (NRHM), Adolescent Division, Ministry of Health and Family Welfare (MoHFW), Government of India 2013 database, anemia in India is a severe grade public health problem with a high prevalence of about $74 \%$ with hemoglobin $<11 \mathrm{gm} / \mathrm{dl}$ [3]. It is widely prevalent in all age groups, nearly $58 \%$ in pregnant women, $50 \%$ among non-pregnant non-lactating women, $56 \%$ among adolescent girls, $30 \%$ in adolescent boys and around $80 \%$ in children under two years of age. The burden of anemia is a major contributor for low birth weight, lowered resistance to infection, poor cognitive and motor development, weakness, fatigue, difficulty in concentrating and lower productivity [3]. Anemia is a multifactorial disease and the magnitude of anemia has significant adverse health, social and economic consequences and demands strategic implementation of prevention and control programmes to treat and prevent anemia. The strategies and comprehensive actions should be community or population group oriented taking into consideration of specific etiologies and local conditions. Seeking for an aim to calculate the total prevalence and disability burden rates in 187 countries over 20 years period (1990-2010), Kassebaum et al. conducted the first comprehensive audit of global burden of anemia based on hemoglobin deficiency. The team enlisted 17 different causes of anemia and the top 5 causes with highest prevalence were iron deficiency anemia (IDA), hookworm, sickle cell disorders (SCD), thalassemias, schistosomiasis and malaria [4]. In Indian scenario, nutritional deficiency, especially iron deficiency is the most common etiological factor for anemia, particularly in adolescent girls when the requirement for iron increases tremendously to physiological need. These adolescent girls who are the future mothers, will have low pre-pregnancy iron reserve to meet the requirement of growing fetus resulting in pre-term babies and/or low birth weight babies. The panel of eminent economists has estimated the benefit-to-cost ratio of iron interventions, cognitive improvement and physical productivity to be as high as 200:1 [3].

As per National Family Health Survey-3 (NFHS-3), nutritional deficiency, in form of malnourishment, anemia, micronutrient deficiency (folate vitamins B12, A, Riboflavin, Thiamin), is highly prevalent in Chhattisgarh state, more so seen in rural community, people with low socioeconomic status, illiterate women and those with low body weight $[5,6]$. Four out of the top 5 causes depicted above by Kasseubaum and team, are quite prevalent in our state. Those are IDA (57.5\%), SCD $(3.2-22.5 \%)$, Beta Thalassemia trait $(0.5-8.5 \%)$ and malaria (8.16\%) $[4,5,7]$. Besides, vitamin B12 (cobalamin) deficiencies are also extremely common in Indian population, owing to vegetarian diet consumed by major population. In most health centers in rural areas, mean corpuscular volume (MCV) is evaluated for macrocytosis and thus used as a screening parameter for macrocytic anemia instead

${ }^{*}$ Corresponding author: Suprava Patel, Department of Biochemistry, All India Institute of Medical Sciences, Raipur, Chhattisgarh, India, Tel: +918518881707; E-mail: dr_suprava@yahoo.co.in

Received April 03, 2017; Accepted April 17, 2017; Published April 24, 2017

Citation: Patel S, Dhuppar P, Bhattar A (2017) Nutritional Anemia Status in Adolescent Girls in Rural Schools of Raipur, India. Med Chem (Los Angeles) 7: 853-856. doi: 10.4172/2161-0444.1000441

Copyright: (c 2017 Patel S, et al. This is an open-access article distributed under the terms of the Creative Commons Attribution License, which permits unrestricted use, distribution, and reproduction in any medium, provided the original author and source are credited. 
of serum vitamin B12 estimation which is an expensive parameter to be used for diagnosing cobalamin deficiency. As a result vitamin B12 deficiency is actually under-diagnosed as MCV alone is an unreliable screening parameter for the same [8]. Raina et al. depicted suboptimal levels of vitamin B12 in 53.6\% of enrolled subjects [9]. Refsum et al. in their study estimated cobalamin deficiency in $75 \%$ of subjects [10]. Present nutritional prophylaxis programme does not include vitamin B12 supplementation which is also a vital micronutrient because it can lead to 'folate trap' and eventually produce megaloblastic anemia. Further reduction in vitamin B12 level leads to hyperhomocysteinemia, which is considered as a risk factor for atherosclerosis. Besides these, early diagnosis and treatment of chronic depletion of cobalamin is crucial failing which it may result in irreversible neurological damage [9].

Compared to vast majority of articles published on prevalence of anemia, mostly IDA, relatively few published data from India are available reflecting vitamin B12 status and its association with anemia in adolescent girls. This study not only would highlight the burden of nutritional anemia in them, but also it would aid in drawing recommendations and rendering suggestions to assess and maneuver the existing schemes.

\section{Aim and Objectives}

1. To estimate the prevalence of anemia among school girls aged $10-18$ years.

2. To associate the severity of anemia with serum levels of ferritin and vitamin B12.

3. To study the association of anemia with age, socio-economic status and BMI.

\section{Materials and Methods}

It was a cross-sectional study that included 382 school going girls of age group $10-18$ years at rural areas of Raipur district, India. All school going adolescent girls of age group 10-18 years whose parents/ LAR signed consent form were included in the study. Those who were not willing to sign, or those with known cases of hemolytic anemia and those beyond the specified age group were not included in the study.

Semi-structured, pre-designed and pre-tested, self-administered questionnaire was used to fill up all required demographic profiles. Blood was collected under all aseptic conditions for evaluating complete hemogram, peripheral smear and sickling test, serum iron, total iron binding capacity (TIBC) and vitamin B12 levels. Serum B12 and Ferritin measured in Electrochemiluminescence method in Cobas e-400 immunoassay autoanalyzer from Roche Diagnostics and serum Iron and TIBC measured by Iron-Ferrozine method in Biosystem B 400 fully automated clinical autoanalyzer from Biosystem reagent and Instruments in the clinical biochemistry laboratory.

Severity of anemia was categorized as given below:

\begin{tabular}{|c|c|}
\hline Severity of anemia & Cut-off $($ WHO criteria) $[11]$ \\
\hline Mild & $10-11.9 \mathrm{gm} / \mathrm{dl}$ \\
\hline Moderate & $7-9.9 \mathrm{gm} / \mathrm{dl}$ \\
\hline Severe & $<7 \mathrm{gm} / \mathrm{dl}$ \\
\hline Vitamin B12 & $<200 \mathrm{pg} / \mathrm{ml}$ \\
\hline Ferritin & $<15 \mathrm{ng} / \mathrm{ml}$ \\
\hline
\end{tabular}

\section{Results}

The results revealed that $36.4 \%$ were found to be anemic in the study group (Table 1). The percentage of mild, moderate and severe anemia in the anemic population was respectively $84.9 \%, 12.9 \%$ and $2.1 \%$ (Table 1 ). The age group of the study population was sub-classified into 10-14 years and 15-18 years taking into consideration that the starting age of menarche is usually 14 years. No significant association of hemoglobin levels could be established with either age or menarche attainment (Table 2 and Figure 1).

The Chi-square test in Tables 3-5 illustrated that hemoglobin concentration was significantly $(\mathrm{p}<0.001)$ associated respectively with the different socioeconomic class, body mass index and diet pattern of the study subjects. Nearly $58 \%$ of adolescent girls had vitamin B12 deficiency. Prevalence of iron deficiency and combined iron and vitamin B12 deficiency was observed to be respectively $11 \%$ and $16.5 \%$ (Figure 2). Serum ferritin levels accorded significant $(\mathrm{p}<0.05)$, between non-anemic and moderately anemic subjects whereas significant

\begin{tabular}{|c|c|c|c|}
\hline $\mathbf{H b} \mathbf{( g m / d \mathbf { l } )}$ & No. Of cases $\mathbf{( n = 3 8 2 )}$ & Percentage & \\
\cline { 1 - 3 } Normal & 243 & 63.6 & \\
\hline Mild & 118 & 30.9 & \\
\cline { 1 - 3 } Moderate & 18 & 4.7 & \multirow{3}{*}{$36.4 \%$} \\
\hline Severe & 3 & 0.8 & \\
\hline Total & 382 & 100 & \\
\hline
\end{tabular}

Table 1: Haemoglobin wise distribution of cases in study group.

\begin{tabular}{|c|c|c|c|c|}
\hline Age group & No. Of cases & Percentage & Anemic & Non-Anemic \\
\hline $10-14$ yrs & 184 & 48.2 & $62(33.6 \%)$ & $122(66.3 \%)$ \\
\hline $15-18 \mathrm{yrs}$ & 198 & 51.8 & $74(37.3 \%)$ & $124(62.6 \%)$ \\
\hline Total & 382 & 100 & 139 & 243 \\
\hline
\end{tabular}

Table 2: Age wise distribution of cases in study group.

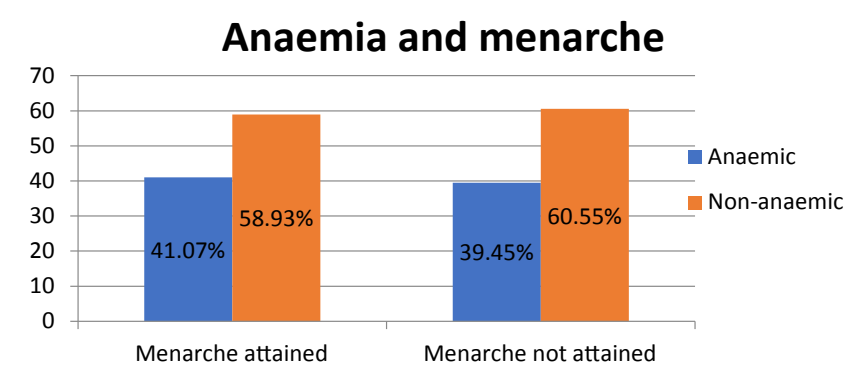

Figure 1: Percentage distribution of attainment of menarche in study population.

\begin{tabular}{|c|c|c|c|c|c|}
\hline \multirow[t]{2}{*}{ SE class } & \multicolumn{4}{|c|}{ Haemoglobin (gm \%) } & \multirow[t]{2}{*}{ Total } \\
\hline & Mild & Moderate & Severe & Normal & \\
\hline II & 5 & 0 & 1 & 12 & 18 \\
\hline III & 46 & 8 & 0 & 86 & 140 \\
\hline IV & 66 & 10 & 2 & 144 & 222 \\
\hline V & 1 & 0 & 0 & 1 & 2 \\
\hline Total & 118 & 18 & 3 & 243 & 382 \\
\hline
\end{tabular}

Chi square test $p<0.001$ highly significant

Table 3: Association between SES and $\mathrm{Hb}$ in study group.

\begin{tabular}{|c|c|c|c|c|c|}
\hline \multirow{2}{*}{ BMI } & \multicolumn{4}{|c|}{ Haemoglobin (gm \%) } & \multirow{2}{*}{ Total } \\
\cline { 2 - 5 } & Mild & Moderate & Severe & Normal & \\
\hline Below 18.5 & 91 & 14 & 2 & 194 & 301 \\
\hline $18.5-25$ & 25 & 4 & 1 & 48 & 78 \\
\hline $25.1-29$ & 2 & 0 & 0 & 1 & 3 \\
\hline Total & 118 & 18 & 3 & 243 & 382 \\
\hline
\end{tabular}

Chi square test $p<0.001$ highly significant

Table 4: Association between BMI and $\mathrm{Hb}$ in study group. 


\begin{tabular}{|c|c|c|c|c|c|}
\hline \multirow{2}{*}{ Diet pattern } & \multicolumn{4}{|c|}{ Haemoglobin (gm \%) } & \multirow{2}{*}{ Total } \\
\cline { 2 - 5 } & Severe & Mild & Moderate & Normal & \\
\hline Vegetarian & 1 & 39 & 3 & 85 & 128 \\
\hline Mixed & 2 & 79 & 15 & 158 & 254 \\
\hline Total & 3 & 118 & 18 & 243 & 382 \\
\hline
\end{tabular}

Chi square test $p<0.001$ highly significant

Table 5: Association between diet pattern and $\mathrm{Hb}$ in study group.

\begin{tabular}{|c|c|c|}
\hline Group & $\begin{array}{l}\text { Serum ferritin (ng/ml) } \\
\quad(\text { mean } \pm \text { SD) }\end{array}$ & $\begin{array}{l}\text { Serum B12 }(\mathrm{pg} / \mathrm{ml}) \\
\text { (mean } \pm \text { SD) }\end{array}$ \\
\hline Normal $^{a}$ & $48.53 \pm 35.71^{* \mathrm{c}}$ & $237.28 \pm 247.56^{* \mathrm{c}}$ \\
\hline Mild Anemia $^{\text {b }}$ & $40.66 \pm 37.19$ & $219.15 \pm 146.22^{* \star c, d}$ \\
\hline Moderate Anemia ${ }^{c}$ & $18.33 \pm 22.21$ & $208.28 \pm 133.23^{\star \star a, d}$ \\
\hline Severe Anemia $^{d}$ & $13.00 \pm 8.88$ & $737.33 \pm 193.53^{* \star \mathrm{a}, \mathrm{c}}$ \\
\hline
\end{tabular}

${ }^{*} p<0.05$ and ${ }^{* *} p<0.001$, when compared to other groups as superscripted

Table 6: Comparison of serum ferritin and B12 levels in different groups of anemia.

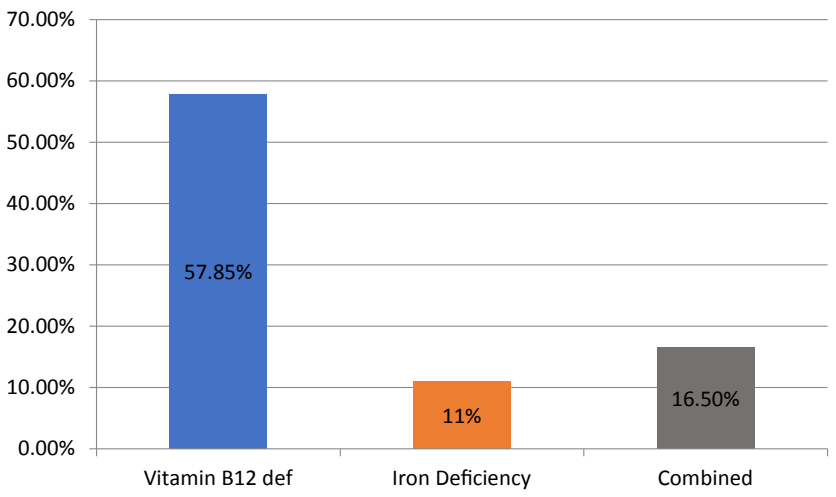

Figure 2: Percentage distribution of Vitamin B12 and iron deficiency in the study population.

differences between the groups were observed for serum vitamin B12 values $(\mathrm{p}<0.001)$ (Table 6).

Hemoglobin concentration correlated positively with serum ferritin $(\mathrm{p}<0.001)$ whereas illustrated no significant correlation with vitamin $\mathrm{B} 12$ ( $\mathrm{p}=0.338)$, as represented in Table 7 . Logistic regression as delineated in Table 8 revealed that anemia was significantly associated with serum ferritin level $(\mathrm{p}<0.01)$ whereas not associated with vegetarian diet, SES and low serum B12 levels. Low serum ferritin $(<15$ $\mathrm{ng} / \mathrm{ml}$ ) attributes to anemia development by 1.93 times.

\section{Discussion}

The study was conducted in the rural schools of Raipur district. The prevalence of anemia in the study group was found to be $36.4 \%$ (Table 1) which corroborated with 2010 global anemia prevalence of $32.9 \%$ as reported by Kassebaum et al. [4]. Approximately $15 \%$ of anemic population represented with moderate to severe degree anemia (Table 1). WHO estimated the prevalence of moderate and severe anemia to be $10 \%$ to $20 \%$ [12]. Main cause for anemia is dietary iron deficiency and many studies have also supported it [13-15]. However, our results decoded for vitamin B12 deficiency being the major concern in adolescent girls. 58\% of the study population represented for vitamin B12 deficiency whereas $11 \%$ had iron deficiency and $16.5 \%$ had combined form (Figure 2). Bhardwaj et al. in their study on adolescent males and females at Kangra, Himachal Pradesh (India), showed anemia in $90 \%$ of the adolescent population enrolled for study

\begin{tabular}{|c|c|c|}
\hline Correlation of Hb with & R value & P value \\
\hline Ferritin & 0.215 & $<0.001^{\text {** }}$ \\
\hline Vitamin B12 & -0.49 & 0.338 \\
\hline
\end{tabular}

Pearsons correlation ${ }^{* *} p<0.001$ highly significant

Table 7: Correlation of $\mathrm{Hb}$ with ferritin and Vitamin B12.

\begin{tabular}{|c|c|c|c|}
\hline Variables & Odds Ratio & $\mathbf{9 5 \%}$ Cl & P value \\
\hline Vegetarian diet & 0.833 & $(0.533-1.30 \mathrm{~s})$ & 0.421 \\
\hline Upper and lower SES & 1.06 & $(0.69-1.62)$ & 0.791 \\
\hline Serum ferritin $<15 \mathrm{ng} / \mathrm{ml}$ & 1.93 & $(1.21-3.08)$ & $0.005^{\star}$ \\
\hline Serum vitamin B12<200 pg/ml & 1.032 & $(0.67-1.57)$ & 0.885 \\
\hline
\end{tabular}

${ }^{*} \mathrm{p}<0.05$ significant

Table 8: Association of sociodemographic parameters with severity of anemia.

and strikingly all adolescent were deficient vitamin B12 levels [16]. The results were also in support with Raina et al. who deciphered vitamin B12 deficiency (sub-optimal or very low) in $78 \%$ adult subjects in the same area [9]. Age and attainment of menarche did not record any significant association in our study that corroborated with the study on adolescent girls in Iran by Ramzi et al. [17]. This could be due to the successful implementation of iron folic acid supplementation to the school going adolescent girls (Table 2; Figure 1). In contrary, few studies had depicted that high prevalence of anemia is associated with attainment of menarche $[18,19]$.

Low hemoglobin concentration recorded significant association with SES, BMI and dietary pattern (Table 3-5) that was found similar to various published studies [20-22]. It causes to be ascribed to cooking practices like overcooking of food, vegetarian diet or even if non-vegetarian, it contained more of animal milk rather than meat. Hemoglobin level documented significantly positive correlation with serum ferritin $(\mathrm{p}<0.001)$ reflects lowering of hemoglobin would result in more release of iron from ferritin, that acts as a reservoir of iron, thus reducing their levels. Low ferritin reservoir $(<15 \mathrm{ng} / \mathrm{ml})$ increase the risk for developing anemia by 1.93 times (Table 8 ). However, hemoglobin was found to posses negative correlation, though not significant, with serum vitamin B12. This could be due to the fact that vitamin B12 is not directly related to red blood cell maturation as that of folic acid. Hence folic acid levels could have directed to a more confirmatory cause for anemia. Basically vitamin B12 have a distinctive role in folate coenzyme synthesis [22]. In this era of folic acid fortification, masking effect of folic acid on vitamin B12 deficiency has become a real concern. Folic acid supplementation to adolescent girls would somehow correct mean corpuscular volume (MCV) and correct anemia but without restoring vitamin B12 level. Uncorrected and delayed diagnosis of vitamin B12 deficiency could effect in irreversible neuropathy [23,24].

\section{Conclusion}

Anemia is considered as a pandemic public health problem. Implementation of supplementation programme is not just enough to treat the endemic due to marked disparities in socioeconomic status, dietary pattern, cultural practices, non-compliance for supplementation and primary health care services. There is need to shift the focus from old paradigm of iron deficiency anemia to other forms of anemia basically vitamin B12 deficiency anemia, as observed from this study. The major limitation of the study is that couldn't evaluate the folic acid status of the adolescent girls due to financial constrain, which could have thrown a confirmatory result regarding anemia status.

\section{Recommendations}

Supplementation with not only iron but also vitamin B12 besides deworming and folic acid is required through national programme. 
Citation: Patel S, Dhuppar P, Bhattar A (2017) Nutritional Anemia Status in Adolescent Girls in Rural Schools of Raipur, India. Med Chem (Los Angeles) 7: 853-856. doi: 10.4172/2161-0444.1000441

\section{Acknowledgements}

The authors acknowledge the immense contribution of all laboratory professionals of Balgopal Children Hospital and Research Center and Department of Biochemistry, All India Institute of Medical Sciences, Raipur, Chhattisgarh in accomplishing this work.

\section{References}

1. WHO (2015) The global prevalence of anaemia in 2011.

2. Kumar A (1999) National nutritional anaemia control programme in India. Indian J Public Health 43: 3-5.

3. Guidelines for control of iron deficiency anemia: Adolescent division, MoH\&FW, Government of India.

4. Kassebaum NJ, Jasrasaria R, Naghavi M, Wulf SK, Johns N, et al. (2014) A systematic analysis of global anemia burden from 1990 to 2010 . Blood 123 615-624.

5. Galhotra A, Padhy G, Pal A, Giri A, Nagarkar N (2014) Mapping the health indicators of Chhattisgarh: A public health perspective. Int J Med Public Health $4: 23$

6. Bentley ME, Griffiths PL (2003) The burden of anemia among women in India Eur J Clin Nutr 57: 52-60.

7. Anaemia, lodine Deficiency and Micro Nutrient Disorders (2013) Press Information Bureau, Government of India, Ministry of Health and Family Welfare.

8. Bhatia P, Kulkarni JD, Pai SA (2012) Vitamin B12 deficiency in India: Mean corpuscular volume is an unreliable screening parameter. Natl Med J India 25: 336-338.

9. Raina S, Sharma K, Chahal J, Kaur N (2014) How common is Vitamin B12 deficiency - A report on deficiency among healthy adults from a medical college in rural area of North-West India. Int J Nutr Pharmacol Neurol Dis 4: 241

10. Refsum H, Yajnik CS, Gadkari M, Schneede J, Vollset SE, et al. (2001) Hyperhomocysteinemia and elevated methylmalonic acid indicate a high prevalence of cobalamin deficiency in Asian Indians. Am J Clin Nutr 74: 233-241.

11. WHO/NMH/NHD/MNM/11.1. Haemoglobin concentration for the diagnosis of anemia and assessment of severity.
12. World Health Organization (1992) The prevalence of anaemia in women: a tabulation of available information.

13. Pandey S, Singh A (2013) A Cross Sectional Study of Nutritional Anemia among Medical Students in a Medical College, at Bilaspur, Chhattisgarh. Natl J Med Res 3: 143-146.

14. Yerpude PN, Jogdand KS (2015) A Cross-Sectional Study to Find Out Prevalence of Anaemia among Adolescent Girls in an Urban Slum Area of South India. Int J Health Sci Res 5: 50-53.

15. Saha J, Sarkar D (2015) Prevalence of iron deficiency and iron deficiency anaemia among nursing students of Bilaspur Chhattisgarh. Int J Med Res Rev 3.

16. Bhardwaj A, Kumar D, Raina SK, Bansal P, Bhushan S, et al. (2013) Rapid Assessment for Coexistence of Vitamin B12 and Iron Deficiency Anemia among Adolescent Males and Females in Northern Himalayan State of India. Anemia.

17. Ramzi M, Haghpanah S, Malekmakan L, Cohan N, Baseri A, et al. (2011) Anemia and iron deficiency in adolescent school girls in kavar urban area, southern iran. Iran Red Crescent Med J 13: 128-133.

18. Leenstra T, Kariuki SK, Kurtis JD, Oloo AJ, Kager PA, et al. (2004) Prevalence and severity of anemia and iron deficiency: cross-sectional studies in adolescent schoolgirls in western Kenya. Eur J Clin Nutr 58: 681-691.

19. Srivastava A, Kumar R, Sharma M (2017) Nutritional anaemia in adolescent girls: an epidemiological study. Int J Community Med Public Health 3: 808-812.

20. Thomas D, Chandra J, Sharma S, Jain A, Pemde HK (2015) Determinants of Nutritional Anemia in Adolescents. Indian Pediatr 52: 867-869.

21. Bharati P, Shome S, Chakrabarty S, Bharati S, Pal M (2009) Burden of anemia and its socioeconomic determinants among adolescent girls in India. Food Nutr Bull 30: 217-226.

22. Perry J, Lumb M, Laundy M, Reynolds EH, Chanarin I (1976) Role of vitamin B12 in folate coenzyme synthesis. Br J Haematol 32: 243-248.

23. Morris MS, Jacques PF, Rosenberg IH, Selhub J (2007) Folate and vitamin B-12 status in relation to anemia, macrocytosis, and cognitive impairment in older Americans in the age of folic acid fortification. Am J Clin Nutr 85: 193-200.

24. Brouwer I, Verhoef $P$ (2007) Folic acid fortification: is masking of vitamin B-12 deficiency what we should really worry about? Am J Clin Nutr 86: 897-898. 